\title{
Kosteneffektivität von Apixaban zur Verhinderung von Schlaganfällen bei Vorhofflimmern in Deutschland
}

\author{
Andreas Reimer • Oliver Schöffski
}

Online publiziert: 12. Februar 2014

(C) The Author(s) 2014. Dieser Artikel ist auf Springerlink.com mit Open Access verfügbar

\begin{abstract}
Background and Objective: Apixaban was shown to be superior to warfarin and acetylsalicylic acid in preventing stroke in patients with atrial fibrillation and at least one additional risk factor. This study compared the cost effectiveness of apixaban versus warfarin and acetylsalicylic acid in this patient population in Germany.

Methods: We constructed a lifetime Markov model with 12 health states, based on the results of the Aristotle and Averroes trial, to evaluate the cost effectiveness of apixaban from the social healthcare perspective. Our base-case assumed a cohort of 65-year old patients with a CHADS 2 Score of 2.1. We used a 1-month cycle-length. The outcome was measured in quality-adjusted life-years (QALYs), costs and incremental-cost-effectiveness ratios (ICER). The robustness of the model was tested with one and two way sensitivity analyses. A probabilistic sensitivity analysis was also performed.

Results: Under base case conditions, total costs per patient were $€ 33,427$ and $€ 33,118$ for apixaban and warfarin. Corresponding QALYs were 10.79 and 10.06 in the lifetime Markov model. The ICER was $€ 423$ per QALY. Monte Carlo simulation demonstrated apixaban was cost-effective in $86 \%$ and $100 \%$ of 10,000 iterations at willingness-to-pay thresholds of $€ 5,000$ and $€ 50,000$ per QALY, respectively. Compared to acetylsalicylic acid or the combination
\end{abstract}

A. Reimer

SLK Kliniken GmbH, Heilbronn, Deutschland

O. Schöffski $(\varangle)$

Lehrstuhl für Gesundheitsmanagement, Friedrich-

Alexander-Universität Erlangen-Nürnberg, Nürnberg,

Deutschland

e-mail: oliver.schoeffski@fau.de of acetylsalicylic acid and clopidogrel apixaban was the economic dominant therapeutic strategy.

Conclusion: The Markov model strongly suggests that apixaban therapy in the German health care setting is a costeffective alternative to warfarin and phenprocoumon, respectively.

\section{Einleitung}

Vorhofflimmern ist die häufigste Rhythmusstörung des Herzens [1]. In Deutschland sind 800.000 Patienten betroffen [1]. Beim Vorhofflimmern handelt es sich um eine Erkrankung des fortgeschrittenen Alters [2]. Für die nächsten Jahrzehnte wird aufgrund der demographischen Entwicklung mit einer Verdoppelung der Patientenzahlen gerechnet [2]. Die Mortalität ist bei Patienten mit Vorhofflimmern als Folge thromboembolischer Ereignisse erhöht [3]. Das Risiko einen ischämen Schlaganfall zu erleiden ist 5-fach erhöht [3]. Aufgrund des hohen thromboembolischen Risikos umfasst die therapeutische Behandlung des Vorhofflimmerns neben der Kontrolle der Herzfrequenz auch die antithrombotische Prophylaxe [4]. Über 5 Jahrzehnte waren die beiden oralen Antikoagulantien Warfarin (Coumadin ${ }^{\circledR}$ ) und Phenprocoumon (Marcumar ${ }^{\circledR}$ ) der Goldstandard in der Prophylaxe thromboembolischer Ereignisse bei Vorhofflimmern. Durch Warfarin wird das Risiko für Schlaganfälle bei Vorhofflimmern um über $60 \%$ reduziert [5]. Den Thrombozytenaggregationshemmern Acetylsalicylsäure (Aspirin ${ }^{\circledR}$ ) und Clopidogrel (Plavix ${ }^{\circledR}$, Iscover ${ }^{\circledR}$ ) sind die oralen Antikoagulantien (Warfarin und Phenprocoumon) klinisch überlegen [6]. Unter „real world Bedingungen“ kommt es bei diesen Produkten jedoch zu zahlreichen therapeutischen Problemen (Wechselwirkungen mit Arzneistoffen und Nahrungsmitteln, pharmakodynamische Variabilität infolge genetischen Polymorphismus, geringe therapeutische Breite) 
bei der Anwendung der oralen Antikoagulantien [7, 8]. In der ambulanten Therapiesituation kommt es durch die praktischen Schwierigkeiten in der Arzneimitteltherapie zu einer Unterversorgung mit den oralen Antikoagulantien Warfarin bzw. Phenprocoumon. In der deutschen MOCA Studie (371 Patienten in 45 Arztpraxen) erhielten $20 \%$ der Patienten - entgegen der Indikation - keine oralen Antikoagulantien [3]. Aufgrund der Schwierigkeiten in der praktischen Durchführung mit Warfarin und Phenprocoumon ist die Arzneistoffgruppe der ",neuen oralen Antikoagulantien“ (NOA) entwickelt worden. Inzwischen haben Dabigatran, Rivaroxaban und Apixaban die EU-weite Zulassung für die Indikation Prophylaxe von Schlaganfällen und systemischen Embolien bei Vorhofflimmern erhalten. Die klinische Wirksamkeit in dieser Indikation von Apixaban wurde in 2 klinischen Studien untersucht. In der doppelblinden, multizentrischen und randomisierten Aristotle Studie (18.021 Patienten) wurde Apixaban mit Warfarin bezüglich der Prävention von Schlaganfällen und systemischen Embolien bei Patienten mit Vorhofflimmern verglichen [9]. Einschlusskriterium war neben Vorhofflimmern zusätzlich mindestens ein weiterer Risikofaktor (Alter über 75 Jahre, stattgefundener Schlaganfall oder TIA, systemische Embolie, Herzinsuffizienz, Diabetes oder Bluthochdruck) [9]. Die Aristotle Studie zeigte, dass Apixaban Warfarin in Bezug auf den primären Endpunkt (hämorrhagischer und ischämer Schlaganfall oder systemische Embolie) signifikant überlegen ist $(1,27 \%$ versus 1,60 \% pro Jahr, Relatives Risiko: 0,79, $95 \%$ Konfidenzintervall: $0,66-0,95, p<0,01$ ) [9]. Apixaban reduziert signifikant die intrakranialen schweren Blutungsereignisse $(0,33 \%$ versus $0,80 \%$ pro Jahr, Hazard Ratio: 0,42 , Konfidenzintervall: $0,30-0,58, p<0,001$ ) [9]. Apixaban wurde darüber hinaus in der doppelblinden, multizentrischen und randomisierten Averroes Studie (5.599 Patienten) geprüft [10]. Die Studie sollte untersuchen, ob Patienten mit Vorhofflimmern, die für Warfarin nicht geeignet sind, mit Apixaban erfolgreich therapiert werden können. Die geprüften Interventionen waren Apixaban $5 \mathrm{mg}$ zweimal täglich und Acetylsalicylsäure (81-324 mg) [10]. In Bezug auf den primären Endpunkt (Schlaganfall oder systemische Embolie) ist Apixaban Acetylsalicylsäure signifikant überlegen (1,6\% versus $3,7 \%$ pro Jahr, $p<0,001)$ [10]. Sowohl im Apixaban- als auch im Acetylsalicylsäurearm treten schwere Blutungsereignisse mit vergleichbarer Häufigkeit auf $(1,4 \%$ versus $1,2 \%$ pro Jahr, $p=0,57$ ) [10].

Obwohl Apixaban aufgrund dieser Studienergebnisse eine valide therapeutische Alternative zu Warfarin und Phenprocoumon darstellt, könnten die höheren Tagestherapiekosten für Apixaban (€ 3,54) im Vergleich zu Phenprocoumon $(€ 0,23)$ eine potentielle Hürde für den verstärkten klinischen Einsatz in Deutschland darstellen. Andererseits berichten Reinhold et al., dass sich die durchschnittlichen Jahreskosten für einen Patienten mit Vorhofflimmern in
Deutschland auf $€ 3.274 \pm 5.134$ belaufen und der wesentlichen Kostentreiber die stationären Kosten sind [11]. Bei Einsatz von Apixaban könnten die höheren Medikamentenkosten durch Reduktion der Folgekosten infolge verringerter Häufigkeit thromboembolischer Ereignisse oder schwerer Blutungsereignisse kompensiert werden. Die vorliegende Studie vergleicht die Kosteneffektivität von Apixaban und Phenprocoumon im deutschen Gesundheitswesen in der Indikation Prophylaxe von Schlaganfällen bei Patienten mit Vorhofflimmern.

\section{Methoden}

\subsection{Studienperspektive und entscheidungsanalytisches Modell}

In der vorliegenden pharmaökonomischen Studie wird die Perspektive der Sozialversicherung gewählt. In der Perspektive der Sozialversicherung werden die Pflegekosten berücksichtigt, die bei der Schlaganfallversorgung $22 \%$ der Gesamtkosten ausmachen [14]. Die Studienpopulation besteht im Base Case aus einer hypothetischen Kohorte 65-jähriger Patienten mit Vorhofflimmern und einem $\mathrm{CHADS}_{2}$ Score von 2,1 (Herzinsuffizienz 1 Punkt; Bluthochdruck 1 Punkt; Alter über 75 Jahre 1 Punkt; Diabetes mellitus 1 Punkt; vorangegangener Schlaganfall oder transistorische Ischäme Attacke 2 Punkte). Zur pharmaökonomischen Analyse der Kosteneffektivität von Apixaban versus Warfarin bei Patienten mit Vorhofflimmern wurde ein Markov Modell entwickelt und in Excel $2007^{\circledR}$ programmiert. Das Markov Modell umfasst 12 Gesundheitszustände: Vorhofflimmern, Ischämer Schlaganfall (temporär), Ischämer Schlaganfall mit schwerer Behinderung (permanent), Ischämer Schlaganfall mit leichter/ohne Behinderung (permanent), Intrakraniale schwere Blutung (temporär), Intrakraniale schwere Blutung mit schwerer Behinderung (permanent), intrakraniale schwere Blutung mit leichter Behinderung (permanent), Extrakraniale schwere Blutung (temporär), Myokardinfarkt (temporär), Wiederholter ischämer Schlaganfall (permanent), Ischämer Schlaganfall und schwere Intrakraniale Blutung (permanent) sowie Tod. Im vorliegenden Modell werden temporäre Gesundheitszustände modelliert um kurzfristige Kosten bzw. Nutzenänderungen abzubilden. Erleidet ein Patient der Kohorte mit Vorhofflimmern einen ischämen Schlaganfall oder eine schwere Intrakraniale Blutung, so ergeben sich bei der akuten stationären Krankenhausversorgung hohe Kosten für die Krankenversicherungen. Lindig et al. zeigen, dass bereits in den ersten 4 Wochen nach dem Schlaganfall $50 \%$ der gesamten Jahreskosten für die GKV entstehen [12]. Daher werden die beiden Gesundheitszustände Ischämer Schlaganfall und Intrakraniale schwere Blutung als temporäre Gesundheitszustände angelegt, während die Folgezustände (Ischämer Schlaganfall 


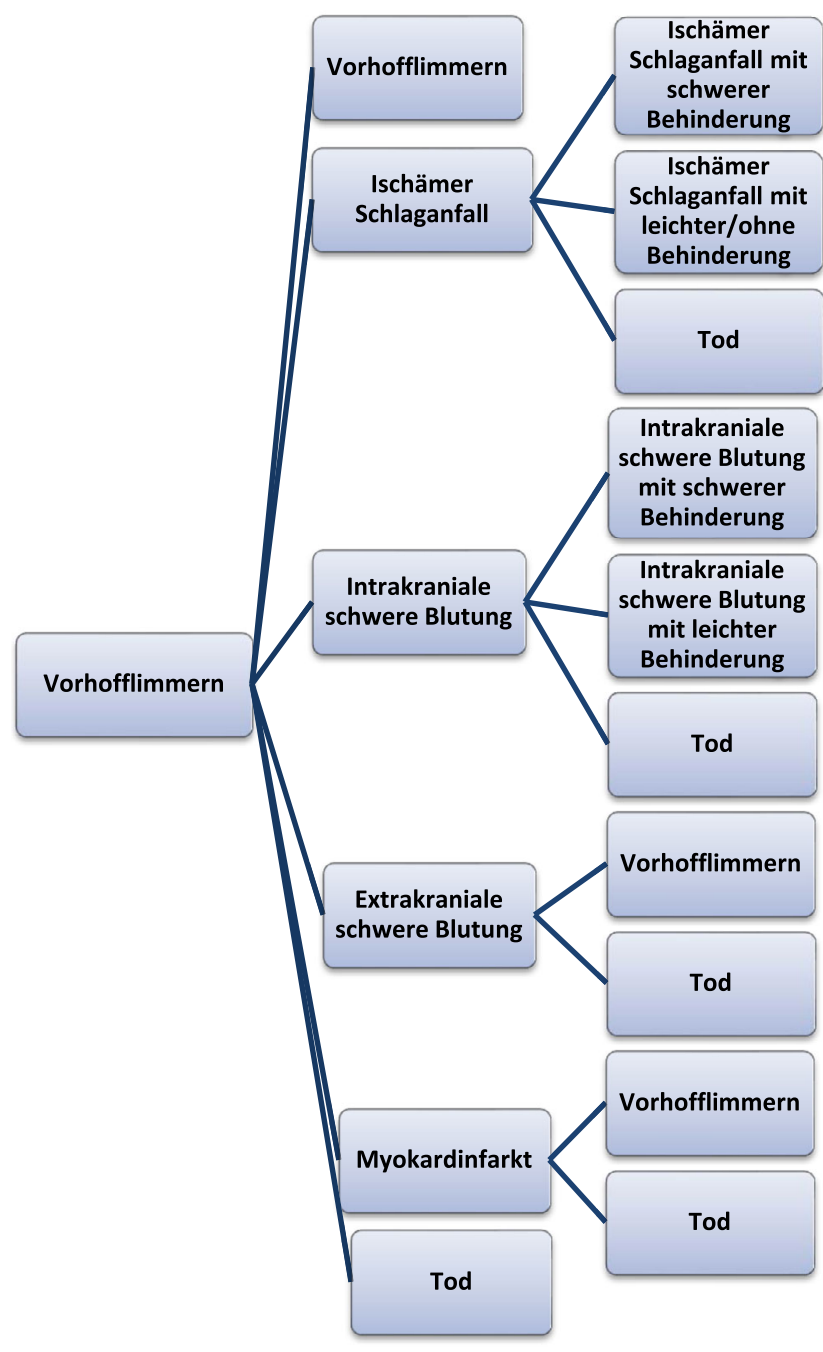

Abb. 1 Schematische Darstellung des Markov Modells

mit schwerer Behinderung, Ischämer Schlaganfall mit leichter/ohne Behinderung sowie Intrakraniale schwere Blutung mit schwerer Behinderung, Intrakraniale schwere Blutung mit leichter Behinderung) als permanente Gesundheitszustände modelliert werden. Der Übergang in diese permanenten Gesundheitszustände ist aus dem Zustand Vorhofflimmern beim Erleiden eines ischämen Schlaganfalls oder eines schweren intrakranialen Blutungsereignis nur über den zugehörigen temporären Gesundheitszustand erreichbar. Auch die Gesundheitszustände Myokardinfarkt bzw. Extrakraniale schwere Blutung sind temporär angelegt. Nach einem Zyklus wechseln alle Patienten der Gesundheitszustände Myokardinfarkt oder Extrakraniale schwere Blutung entweder in den Zustand Vorhofflimmern oder Tod (Abb. 1).

Das primäre Szenario sind die Therapieoptionen Apixaban und Warfarin bei Vorhofflimmern gemäß der Aristotle Studie. Kommt ein Patient für die Therapie mit Warfarin nicht in Betracht, da er beispielsweise nicht stabil in den INR (international normalized ratio) Zielbereich 2-3 ein- stellbar ist, können weitere therapeutische Optionen erwogen werden. Vor Einführung der neuen oralen Antikoagulantien (Dabigatran, Rivaroxaban und Apixaban) waren dies Acetylsalicylsäure und die Kombination aus Acetylsalicylsäure und Clopidogrel. Apixaban soll auch gegen diese beiden Therapieoptionen pharmaökonomisch evaluiert werden. Die Zykluslänge beträgt bei allen Szenarien einen Monat. Innerhalb eines Monats ist die Wahrscheinlichkeit zwei relevante klinische Ereignisse zu erleiden gering. Im Base Case wird eine Diskontierungsrate von $3 \%$ verwendet. Kosten und Nutzen werden mit der gleichen Diskontierungsrate diskontiert.

\subsection{Modell Inputs}

Zur Berechnung der QALYs werden die Nutzwerte für die im vorliegenden Markov Modell relevanten Gesundheitszustände der Publikation von Gage et al. entnommen [13]. Für jeden Zyklus wird die Summe aus den QALYs der verschiedenen Gesundheitszustände berechnet. Die QALYs werden gebildet durch Multiplikation der Nutzwerte mit dem Anteil der Patientenkohorte in den jeweiligen Gesundheitszuständen. Anschließend werden die QALYs über alle $\mathrm{Zy}$ klen aufsummiert. Zur Ermittlung der Zykluskosten für die im Modell angelegten Gesundheitszustände wird auf Daten von Milde zurückgegriffen [14]. In der Arbeit von Milde werden aus der Perspektive der Sozialversicherung die Kosten für die Schlaganfallversorgung evaluiert. Die Versorgungskosten aus der Perspektive der Sozialversicherung für ischämen Schlaganfall betragen nach einem Jahr $€ 17.020$ und nach zwei Jahren $€ 24.621$. Die Versorgungskosten aus der Perspektive der Sozialversicherung für eine intrakraniale schwere Blutung betragen nach einem Jahr $€ 24.246$ und nach zwei Jahren $€$ 31.779. Zur Festlegung der Ereigniskosten für eine schwere extrakraniale Blutung wird auf Kostendaten gastrointestinaler Blutungen zurückgegriffen, die durch Phenprocoumon verursacht worden sind. Gastrointestinale Blutungen sind die häufigsten schweren extrakranialen Blutungen. In Schwebe et al. wurden die stationären Kosten von gastrointestinalen Blutungen ermittelt, die als unerwünschte Arzneimittelwirkung der Phenprocoumon Therapie auftraten [15]. Kristiansen et al. haben die Austauschbarkeit von Phenprocoumon und Warfarin hinsichtlich Nebenwirkungen und Wirksamkeit gezeigt [16]. Die Kosten für durch Phenprocoumon verursachte gastrointestinale Blutungen werden daher als vergleichbar mit den von Warfarin verursachten Kosten extrakranialer schwerer Blutungen angesehen. Die Kosten eines Myokardinfarkts werden der Arbeit von Tieman entnommen [17]. Die Kosten für die in den verschiedenen Therapieoptionen verwendeten Arzneimittel werden der Roten Liste ${ }^{\circledR}$ entnommen [18]. In der Roten Liste ${ }^{\circledR}$ werden die Kosten als Apothekenverkaufspreise inklusive Umsatzsteuer ausgewiesen. Der GKV 
Tab. 1 Kostenvariablen (Base Case) mit Wertebereich für Sensitivitätsanalysen

\begin{tabular}{|c|c|c|c|}
\hline \multirow[t]{2}{*}{ Arzneimittel } & \multicolumn{3}{|c|}{ Kosten Arzneimittel: Monatskosten in $€$} \\
\hline & Monatskosten & Wertebereich & Quelle \\
\hline Warfarin (beinhaltet auch INR Monitoring) & 50 & $(25-75)$ & [18]/Eigene Berechnungen \\
\hline Apixaban & 99 & $(50-150)$ & [18]/Eigene Berechnungen \\
\hline Acetylsalicylsäure & 1 & & [18]/Eigene Berechnungen \\
\hline Clopidogrel + Acetylsalicylsäure & 36 & & [18]/Eigene Berechnungen \\
\hline \multirow[t]{2}{*}{ Gesundheitszustand } & \multicolumn{3}{|c|}{ Kosten Gesundheitszustände: Ereigniskosten/Monatskosten in $€$} \\
\hline & Kosten & Wertebereich & Quelle \\
\hline Intakraniale schwere Blutung & Ereigniskosten: 9.626 & $(4.813-14.439)$ & {$[14]$} \\
\hline Intrakraniale schwere Blutung mit schwerer Behinderung & Monatskosten: 1.257 & $(629-1.886)$ & {$[14] /$ Eigene Berechnungen } \\
\hline Intrakraniale schwere Blutung mit leichter Behinderung & Monatskosten: 859 & $(430-1.228)$ & [14]/Eigene Berechnungen \\
\hline Ischämer Schlaganfall & Ereigniskosten: 7.690 & $(3.845-11.535)$ & [14]/Eigene Berechnungen \\
\hline Ischämer Schlaganfall mit schwerer Behinderung & Monatskosten: 1.075 & $(538-1.613)$ & {$[14] /$ Eigene Berechnungen } \\
\hline Ischämer Schlaganfall mit leichter/ohne Behinderung & Monatskosten: 674 & $(337-1.011)$ & {$[14] /$ Eigene Berechnungen } \\
\hline Wiederholter ischämer Schlaganfall & Monatskosten: 1.770 & $(885-2.665)$ & [14]/Eigene Berechnungen \\
\hline Ischämer Schlaganfall und schwere intrakraniale Blutung & Monatskosten: 1.770 & $(885-2.665)$ & [14]/Eigene Berechnungen \\
\hline Myokardinfarkt & Ereigniskosten: 3.102 & $(1.553-4.653)$ & [17]/Eigene Berechnungen \\
\hline Extrakraniale schwere Blutung & Ereigniskosten: 3.053 & $(1.527-4.580)$ & {$[15] /$ Eigene Berechnungen } \\
\hline $\begin{array}{l}\text { Tod (nur bei Versterben des Patienten nach Ischämer } \\
\text { Schlaganfall (temporär)/nach Intrakranialer schwerer } \\
\text { Blutung (temporär) im anschließenden } \\
\text { Behandlungsprozess }\end{array}$ & Ereigniskosten: 9.675 & $(4.823-14.498)$ & [14]/Eigene Berechnungen \\
\hline
\end{tabular}

müssen jedoch vom Apothekenverkaufspreis Preisnachlässe gewährt werden. Dazu gehören die Rabatte Herstellerrabatt und Apothekenrabatt sowie die Patientenzuzahlung. Die für die GKV letztlich relevanten Kosten ergeben sich, wenn der Apothekenverkaufspreis um diese gesetzlich verankerten Preisnachlässe vermindert wird. Bei den Kosten für die Warfarin Therapie werden zudem die Kosten für ein ambulantes ärztliches INR Monitoring (alle 4 Wochen) und eine Packung CoaguChek Teststreifen berücksichtigt (Tab. 1).

Für die medikamentenbezogenen Übergangswahrscheinlichkeiten im Base Case wird auf die in den betreffenden Studien (Aristotle, Averroes, Active A) publizierten Ereignisraten für ischämen Schlaganfall, intrakraniale und extrakraniale schwere Blutung und Myokardinfarkt zurückgegriffen. $\mathrm{Zu}$ Beginn der Simulation befindet sich die gesamte Kohorte im Gesundheitszustand Vorhofflimmern und weist einen durchschnittlichen $\mathrm{CHADS}_{2}$ Score von 2,1 auf. Erleidet nun ein Patient der Kohorte einen ischämen Schlaganfall oder eine intrakraniale schwere Blutung so nimmt das Risiko für ein weiteres thromboembolisches oder hämorrhagisches Ereignis zu. Folglich erhöht sich für Patienten in den Gesundheitszuständen Ischämer Schlaganfall mit leichter/ohne bzw. schwerer Behinderung oder Intrakraniale schwere Blutung mit leichter bzw. schwerer Behinderung der $\mathrm{CHADS}_{2}$ Risikoscore auf $\geq 3$. Um die thromboembo- lischen und hämorrhagischen Ereignisraten von Patienten nach bereits erlittenem ischämen Schlaganfall oder intrakranialer schwerer Blutung abzuschätzen wird auf Subgruppenanalysen $\left(\mathrm{CHADS}_{2}\right.$ Risikoscore $\left.\geq 3\right)$ zurückgegriffen. Die Übergangswahrscheinlichkeiten werden in Abhängigkeit der Anzahl durchlaufener Zyklen modelliert, da mit zunehmendem Alter der Patienten die Wahrscheinlichkeit steigt unter Therapie mit oralen Antikoagulantien einen ischämen Schlaganfall zu erleiden. Beim ischämen Schlaganfall wird angenommen, dass das ischäme Schlaganfallrisiko mit jeder Lebensdekade um das 1,4-fache zunimmt [19]. Für intrakraniale schwere Blutungen wird eine Zunahme des Risikos um das 1,97-fache und bei Herzinfarkt um das 1,3-fache pro Lebensjahrzehnt im Modell implementiert [19]. Patienten können im vorliegenden Modell aufgrund des Vorhofflimmerns und den damit verbundenen klinischen Ereignissen versterben. Andererseits können Patienten auch versterben durch krankheitsunspezifische Ursachen, die nicht auf das Vorhofflimmern zurückzuführen sind. Diese Hintergrundmortalität wird im vorliegenden Modell aus Sterbetafeln des Bundesamtes für Statistik entnommen [20]. Die Hintergrundmortalität wird mit 1,3 multipliziert, um die bei Vorhofflimmern erhöhte Sterblichkeit im Modell abzubilden [19]. Nach Erleiden eines ischämen Schlaganfalls oder eines schweren intrakranialen Blutungsereignisses erhöht sich der Multiplikationsfaktor auf 2,3 [19]. Für die Ge- 
Tab. 2 Modellvariablen (Base Case) mit Wertebereich für Sensitivitätsanalysen

\begin{tabular}{|c|c|c|c|}
\hline Gesundheitsereignis & Medikament & Jährliche Rate & Quelle \\
\hline \multirow[t]{4}{*}{ Ischämer Schlaganfall unter: } & Apixaban & $0,97 \%$ & [9] \\
\hline & Warfarin & $1,05 \%$ & [9] \\
\hline & Acetylsalicylsäure & $3,0 \%$ & {$[10]$} \\
\hline & Acetylsalicylsäure + Clopidogrel & $1,9 \%$ & {$[23]$} \\
\hline \multirow[t]{4}{*}{ Intrakraniales schweres Blutungsereignis unter: } & Apixaban & $0,33 \%$ & [9] \\
\hline & Warfarin & $0,80 \%$ & [9] \\
\hline & Acetylsalicylsäure & $0,40 \%$ & {$[10]$} \\
\hline & Acetylsalicylsäure + Clopidogrel & $0,40 \%$ & {$[23]$} \\
\hline \multirow[t]{4}{*}{ Extrakraniales schweres Blutungsereignis unter: } & Apixaban & $1,79 \%$ & [9] \\
\hline & Warfarin & $2,27 \%$ & [9] \\
\hline & Acetylsalicylsäure & $0,90 \%$ & {$[10]$} \\
\hline & Acetylsalicylsäure + Clopidogrel & $1,60 \%$ & {$[23]$} \\
\hline Anteil & Prozentwert & Wertebereich & Quelle \\
\hline \multicolumn{4}{|c|}{ Anteil ischämer Schlaganfälle unter Apixaban/Warfarin } \\
\hline Tödlich & $8,20 \%$ & $8,2-10,1 \%$ & [19] \\
\hline Mit schwerer Behinderung & $40,2 \%$ & $40,2-41,7 \%$ & [19] \\
\hline Mit leichter/ohne Behinderung & $51,6 \%$ & $43,9-55,8 \%$ & [19] \\
\hline \multicolumn{4}{|c|}{ Anteil ischämer Schlaganfälle unter Acetylsalicylsäure } \\
\hline Tödlich & $17,9 \%$ & $10,1-17,9 \%$ & {$[22]$} \\
\hline Mit schwerer Behinderung & $30,0 \%$ & $30,0-41,1 \%$ & {$[22]$} \\
\hline Mit leichter/ohne Behinderung & $52,1 \%$ & $35,8-54,3 \%$ & {$[22]$} \\
\hline \multicolumn{4}{|c|}{ Anteil ischämer Schlaganfälle unter Acetylsalicylsäure + Clopidogrel } \\
\hline Tödlich & $23,20 \%$ & $20,2-26,4 \%$ & [24] \\
\hline Mit schwerer Behinderung & $21,90 \%$ & $18,0-25,0 \%$ & {$[24]$} \\
\hline Mit leichter/ohne Behinderung & $54,90 \%$ & - & [24] \\
\hline \multicolumn{4}{|c|}{ Anteil intrakranialer schwerer Blutungsereignisse unter Apixaban, Warfarin, Acetylsalicylsäure, Acetylsalicylsäure und Clopidogrel } \\
\hline Tödlich & $36,4 \%$ & $28,3-45,2 \%$ & [19] \\
\hline Mit schwerer Behinderung & $14,10 \%$ & $9,0-21,4 \%$ & [19] \\
\hline Mit leichter Behinderung & $49,5 \%$ & - & [19] \\
\hline \multicolumn{4}{|c|}{ Anteil extrakranialer schwerer Blutungsereignisse unter Apixaban, Warfarin, Acetylsalicylsäure, Acetylsalicylsäure und Clopidogrel } \\
\hline Tödlich & $5,10 \%$ & & [25] \\
\hline \multicolumn{4}{|c|}{ Anteil Myokardinfarkt unter Apixaban, Warfarin, Acetylsalicylsäure, Acetylsalicylsäure und Clopidogrel } \\
\hline Tödlich & $28,40 \%$ & & [26] \\
\hline
\end{tabular}

sundheitszustände wiederholter Ischämer Schlaganfall bzw. Ischämer Schlaganfall und schwere Intrakraniale Blutung wird eine Übersterblichkeit gegenüber der Hintergrundmortalität von $8 \%$ pro Jahr modelliert [21]. Die in der Aristotle Studie für Apixaban gezeigte geringere Mortalität gegenüber Warfarin wird nicht direkt modelliert. Die geringere Sterblichkeit der mit Apixaban behandelten Patienten ergibt sich indirekt aus der Häufigkeit der klinischen Ereignisse und deren krankheitsspezifischen Mortalität (Tab. 2).

\subsection{Modellannahmen}

Die Wirksamkeit der pharmakoökonomisch evaluierten Arzneimittel Apixaban, Warfarin, Acetylsalicylsäure und die Kombinationstherapie aus Acetylsalicylsäure und Clopidogrel wird über den Zeitraum der klinischen Studie hinaus prolongiert. So wird beispielsweise davon ausgegangen, dass Apixaban auch nach 35 Therapiejahren deutlich weniger schwere Blutungsereignisse hervorruft als War- 
farin, obgleich das Studien Follow up der Aristotle Studie nur 1,8 Jahre betrug. Für den Myokardinfarkt wird im Base Case die jährliche Ereignisrate von Apixaban auch für Warfarin, Acetylsalicylsäure sowie Acetylsalicylsäure und Clopidogrel herangezogen. Aufgrund der mangelnden statistischen Signifikanz wird in den relevanten Studien (Aristotle, Averroes, Active-A) auch für das Markov Modell bei allen 3 Vergleichsinterventionen von der gleichen Übergangswahrscheinlichkeit vom Gesundheitszustand Vorhofflimmern nach Myokardinfarkt ausgegangen. Bei Patienten der Kohorte, die im Verlauf ein schweres intrakraniales Blutungsereignis erleiden, wird die dauerhafte Umstellung der Therapie auf Acetylsalicylsäure simuliert. Bei einem extrakranialen schweren Blutungsereignis erfolgt die Umstellung nur für einen Zyklus. Bei der Evaluation von Acetylsalicylsäure und Clopidogrel wird hingegen angenommen, dass auch nach einem schweren Blutungsereignis die Therapie mit der Kombination aus Acetylsalicylsäure und Clopidogrel fortgesetzt wird. Das Erleiden eines Herzinfarkts führt in dem vorliegenden Modell nicht zu einem dauerhaften Gesundheitszustand mit größerer Behinderung. Das gleiche trifft auch für ein extrakraniales schweres Blutungsereignis zu. Der Übergang aus einem permanenten Zustand mit geringerer Lebensqualität in einen Zustand mit höherer Lebensqualität ist nicht zugelassen.

\subsection{Sensitivitätsanalysen und Subgruppenanalysen}

Univariate Sensitivitätsanalysen wurden für folgende Modellvariablen durchgeführt: Kostenparameter, Nutzwerte, Übergangswahrscheinlichkeiten und Diskontierungsrate. Parameter können jedoch auch gegenseitig voneinander abhängen und die Inkrementelle Kosten-Nutzen Relation (IKER) beeinflussen. Die Ereignisraten für Ischämen Schlaganfall und Intrakraniale schwere Blutung wurden in einer bivariaten Sensitivitätsanalyse über das Konfidenzintervall simultan verändert, um deren gemeinsamen Einfluss auf das pharmaökonomische Ergebnis zu zeigen. $\mathrm{Zu}$ sätzlich wurde eine 10.000 Iterationen umfassende Monte Carlo Simulation durchgeführt, um gegenseitige Abhängigkeit der Modellparameter zu untersuchen. Der Monte Carlo Simulation wurde eine triangulare Verteilung der Modellparameter zugrunde gelegt. Durch die Annahme einer triangularen Verteilung wird den spezifischen Eigenschaften der Input Variablen Rechnung getragen (klinische Ereigniskosten bzw. Arzneimittelkosten können beispielsweise nie kleiner $€ 0$ sein). Darüber hinaus wird für verschiedene Subgruppen (Patienten mit höherem ischämen Schlaganfallrisiko, Patienten mit höherem Blutungsrisiko sowie Patienten mit Niereninsuffizienz) die IKER berechnet.
Tab. 3 Bivariate Sensitivitätsanalyse

\begin{tabular}{llllll}
\hline & \multicolumn{5}{c}{ Hazard Ratio ischämer Schlaganfall } \\
\cline { 2 - 5 } & & 0.74 & 0.87 & 1 & 1.13 \\
\hline Hazard Ratio & 0.58 & $<0$ & $€ 3.259$ & $€ 7.835$ & $€ 14.496$ \\
Intrakraniale & 0.49 & $<0$ & $€ 1.231$ & $€ 4.362$ & $€ 8.543$ \\
Blutung & 0.39 & $<0$ & $<0$ & $€ 1.068$ & $€ 3.340$ \\
& 0.3 & $<0$ & $<0$ & $<0$ & $<0$ \\
\hline
\end{tabular}

\section{Ergebnisse}

Unter Base Case Bedingungen können bei Patienten mit Vorhofflimmern (Risiko: CHADS $_{2}$ Score von 2,1) unter Apixaban Therapie 10,79 QALYs generiert werden zu durchschnittlichen Kosten von $€ 33$.427. Wird das gleiche Patientengut mit Warfarin behandelt, ergibt sich eine durchschnittliche Lebenszeit von 10,06 QALYs und durchschnittliche Kosten von $€$ 33.118. Die inkrementelle Kosteneffektivitätsrelation Apixaban versus Warfarin beträgt $€ 423$ pro QALY. Die Kombinationstherapie aus den Thrombozytenaggregationshemmern Acetylsalicylsäure und Clopidogrel ergibt bei Kosten von $€ 34.547$ 9,63 QALYs, während in der Monotherapie mit Acetylsalicylsäure die Patienten durschnittlich 8,88 QALYs leben zu durchschnittlichen Kosten von $€$ 40.657. Sowohl Kombinations(Acetylsalicylsäure und Clopidogrel) als auch Monotherapie (Acetylsalicylsäure) werden von Apixaban ökonomisch dominiert. Das Modell ist sensitiv im Bezug auf die Arzneimittelkosten von Apixaban. Bei Apixaban Monatskosten von $€ 150$ ergibt sich eine IKER von $€ 9.551$ pro QALY. Bei Monatskosten von $€ 50$ ist Apixaban gegenüber Warfarin die ökonomisch dominante Therapiestrategie. Wie die bivariaten Sensitivitätsanalyse zeigt, wird die Therapieoption Apixaban gegenüber Warfarin pharmaökonomisch weniger vorteilhaft, wenn der Hazard Ratio von ischämen Schlaganfall und/oder intrakranialer schwerer Blutung ansteigen (Tab. 3).

Aus der probabilistischen Sensitivitätsanalyse geht hervor, dass Apixaban bezogen auf eine Grenze der Zahlungsbereitschaft von $€ 5.000$ pro QALY in $85,99 \%$ der 10.000 Iterationen gegenüber Warfarin die kosteneffektivere Alternative ist. Bezogen auf eine Zahlungsbereitschaft von $€ 50.000$ pro QALY ist Apixaban in $100 \%$ der 10.000 Iterationen die kosteneffektivere Therapiestrategie (Abb. 2).

Bei der Subgruppenanalyse nach dem CHADS$_{2}$ Score ergibt sich bei einem Patientengut mit niedrigerem Risikoprofil $\left(\mathrm{CHADS}_{2}\right.$ Score von 1) eine IKER von $€ 3.673$ pro QALY. Bei Patienten mit höherem Risiko $\left(\mathrm{CHADS}_{2}\right.$ Score von 3) wird Warfarin durch Apixaban ökonomisch dominiert. Ebenso ist Apixaban bei Patienten mit steigendem Blutungsrisiko (HAS-BLED Score $\geq 2$ ) gegenüber Warfarin die ökonomisch dominante Strategie. Sowohl bei Patienten 


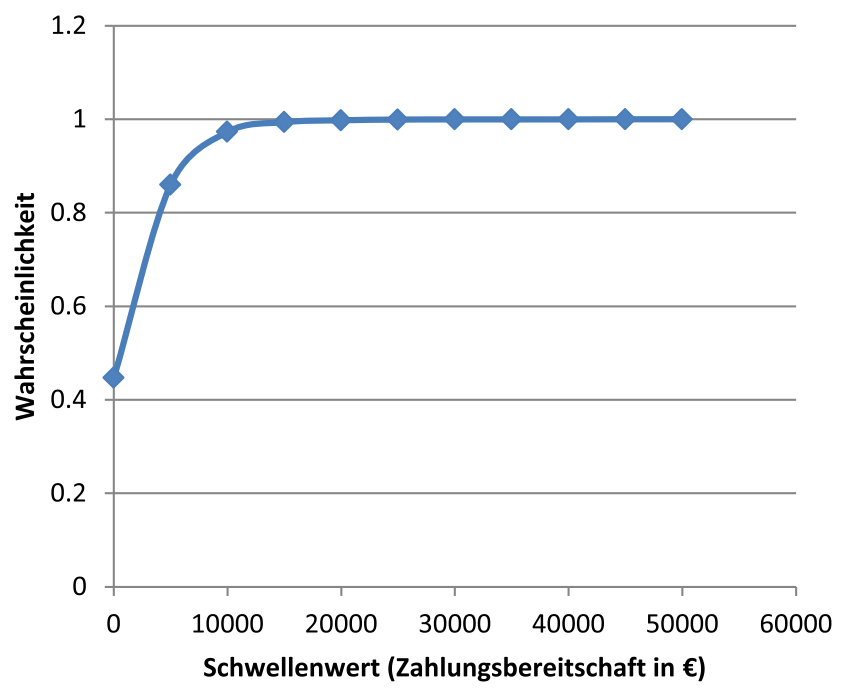

Abb. 2 Kosteneffektivitäts-Akzeptabilitätskurve

mit milder als auch moderater bis schwerer Niereninsuffizienz wird Warfarin durch Apixaban ökonomisch dominiert.

\section{Diskussion}

Die Ergebnisse der vorliegenden pharmaökonomischen Evaluation zeigen, dass mit Apixaban bei Patienten mit Vorhofflimmern und $\mathrm{CHADS}_{2}$ Score von 2,1 gegenüber Warfarin mehr QALYS (10,79 QALYs versus 10,06 QALYs) generiert werden können. Darüberhinaus ergibt die Simulation im Lebenszeit Markov Modell, dass in Bezug auf die erzielbaren QALYs Apixaban auch den Alternativen Kombitherapie Acetylsalicylsäure und Clopidogrel (9,63 QALYs) sowie Acetylsalicylsäure (8,88 QALYs) überlegen ist. Acetylsalicylsäure schneidet bei Patienten mit diesem Risikoprofil bezogen auf die erreichbaren QALYs am schlechtesten ab. Außerdem reduziert Apixaban gegenüber Warfarin die Kosten für die eintretenden klinischen Ereignisse aufgrund der erheblichen Verminderung der intrakranialen schweren Blutungen. Bei Apixaban Monatskosten von $€ 99$ beträgt die IKER Apixaban/Warfarin $423 €$ pro QALY. Das Modell zeigt sich gegenüber einer Vielzahl von Variationen der Input Parameter robust. Auf Veränderungen der Arzneimittelkosten für Apixaban und der Wahrscheinlichkeit für intrakraniale schwere Blutungen sowie ischäme Schlaganfälle reagiert das Modell sensitiv. Die starke Abhängigkeit der IKER Apixaban/Warfarin von den Apixaban Monatskosten steht in Einklang mit Sensitivitätsanalysen weiterer pharmaökonomischer Publikationen zu Apixaban. So zeigen beispielsweise Lee et al., dass bei einem Apixaban Monatspreis von \$ 102 Apixaban gegenüber Warfarin die dominante Strategie ist [19]. Bei einem Apixaban Monatspreis von \$ 308 beträgt die IKER Apixaban/Warfarin \$ 35.583 pro QALY [19].
Die Betrachtung unterschiedlicher Patientensubgruppen unterstützt die Kosteneffektivität von Apixaban. Apixaban ist ab einem $\mathrm{CHADS}_{2}$ Score $\geq 3$ oder einem HAS-BLED Score $\geq 2$ die dominante Strategie gegenüber Warfarin. Der vorliegenden Kosteneffektivitätsuntersuchung sind bereits einige pharmaökonomische Studien aus der Perspektive des jeweiligen nationalen Gesundheitssystems vorangegangen. Lee et al. zeigten mittels eines Lebenszeit Markov Modell (35 Jahre), dass Apixaban gegenüber Warfarin die dominante Strategie ist [19]. In der Base Case Analyse ergaben sich für einen 65-jährigen Patienten (CHADS $_{2}$ Score von 2,1) unter Therapie mit Apixaban 11,16 QALYs und mit Warfarin 10,69 QALYs [19]. Die zugehörigen Kosten beliefen sich für Apixaban auf \$86.007 und \$ 94.941 für Warfarin [19]. Damit wurden unter Apixaban mehr QALYs zu geringeren Gesamtkosten akkumuliert. Die Ergebnisse waren sensitiv in Bezug auf die Kosten für Apixaban und alle Parameter, die sich auf die Häufigkeit intrakranialer schwerer Blutungen unter Therapie mit Apixaban bzw. Warfarin beziehen [19]. Da die Preisbildung zum Zeitpunkt der Arbeit von Lee et al. für Apixaban noch nicht abgeschlossen war, wurde ein dem Dabigatran vergleichbarer Tagestherapiepreis von $\$ 6,78$ im Base Case angesetzt [19]. Kamel et al. untersuchten die Kosteneffektivität von Apixaban versus Warfarin in der Sekundärprophylaxe von Schlaganfällen infolge von Vorhofflimmern [28]. Sie verfolgten in ihrem Markov Modell eine hypothetische Kohorte 70-jähriger Patienten über 20 Jahre [27]. Durch die Therapie mit Apixaban konnten zusätzlich 0,28 QALY generiert werden zu Kosten von \$3.200. Damit ergab sich eine IKER von \$ 11.400 pro QALY [27]. In der Arbeit von Kamel et al. wurde auf den damaligen Preis von Apixaban in Europa zurückgegriffen. Apixaban war bis \$ 11,6 Tagestherapiekosten kosteneffektiv (IKER $<\$ 50.000$ pro QALY) und ab \$ 5,66 oder weniger die pharmaökonomisch dominante Strategie [27]. Lee et al. untersuchten auch die Kosteneffektivität von Apixaban gegenüber Acetylsalicylsäure [22]. Sie berichteten, dass bezogen auf einen Zeitraum von 10 Jahren Apixaban gegenüber Acetylsalicylsäure die pharmaökonomisch dominante Strategie ist [22]. Während bei der Apixaban Therapie Gesamtkosten von \$ 44.232 entstehen, sind es unter Acetylsalicylsäure \$ 50.066 [22]. Gleichzeitig wurden mit Apixaban 6,87 QALYs und mit Acetylsalicylsäure 6,51 QALYs erreicht [22]. Der pharmaökonomischen Untersuchung lag die Simulation in einem Markov Modell mit einer hypothetische Kohorte von 70jährigen Patienten CHADS $_{2}$ Score von 2) zugrunde [22]. Mit der vorliegenden Kosteneffektivitätsuntersuchung kann die Preisbildung von Apixaban im Kontext des deutschen Gesundheitswesens erstmals bewertet werden. Die langfristigen ökonomischen Konsequenzen der Therapieentscheidungen zwischen Apixaban und Warfarin bzw. Phenprocoumon werden dadurch abschätzbar. Bei der Bewertung der 
Ergebnisse der hier vorliegenden pharmaökonomischen Studie müssen allerdings Annahmen und Limitationen des Modells berücksichtigt werden. Die wichtigste Annahme ist, dass die therapeutische Überlegenheit von Apixaban gegenüber Warfarin bzw. Phenprocoumon in Bezug auf die schweren Blutungsereignisse und insbesondere die intrakranialen Blutungen in einen wesentlich längeren Zeitraum extrapoliert werden kann. Die durchschnittliche Studienpopulation könnte sich in wichtigen Begleiterkrankungen, die für den weiteren Krankheitsverlauf entscheidend sind, von der tatsächlichen Patientenpopulation in Deutschland unterscheiden. Die ökonomischen Konsequenzen einer möglicherweise schlechteren Compliance bei der Therapie mit Apixaban unter „real world Bedingungen“ bleiben unberücksichtigt. So muss Apixaban im Gegensatz zu Warfarin zweimal täglich gegeben werden. Wenn eine Dosis vergessen wird, so ist aufgrund der kurzen Halbwertszeit von Apixaban die Gefahr für ein thromboembolisches Ereignis größer. Ein weiterer kritischer Punkt der pharmaökonomischen Analyse sind die Kosten der Warfarin bzw. Phenprocoumon Therapie. Die Kosten setzen sich zusammen aus den Kosten für das Arzneimittel und das Monitoring der Therapie (INR Messung). In der vorliegenden Arbeit werden die Jahreskosten der Warfarin Therapie mit $€ 600$ angesetzt. Dies ist verglichen mit anderen europäischen Ländern ein durchschnittlicher Wert. So geben Gonzalez-Juanatey et al. die Kosten für das INR Monitoring (ohne Arzneimittelkosten) in Spanien mit $€ 383$ (gut einstellbare Patienten) bis $€ 473$ (schlecht einstellbare Patienten) an [28]. Eine schwedische KostenEffektivitätsuntersuchung zu Dabigatran berichtet Kosten der Warfarin Therapie von $€ 776$ pro Jahr [29]. In Deutschland ist nicht Warfarin, sondern Phenprocoumon das bevorzugte orale Antikoagulans. Da von Kristiansen et al. [16]. die Austauschbarkeit von Warfarin und Phenprocoumon gezeigt wurde, lassen sich die Ergebnisse der pharmaökonomischen Untersuchung auf Phenprocoumon übertragen. Zudem zeigt eine kürzlich publizierte Studie von Amin et al., dass sich die Ergebnisse der Aristotle Studie auch auf ,real world" Patienten mit Behandlungsdauer über den Studienzeitraum hinaus übertragen lassen [30]. Dadurch scheint die Grundannahme des Modells plausibel, dass sich die klinische und ökonomische Überlegenheit von Apixaban gegenüber Warfarin nicht auf den Studienzeitraum beschränkt.

\section{Schlussfolgerung}

Apixaban ist, aus der Sozialversicherungsperspektive betrachtet, eine kosteneffektive Alternative zu den bisher etablierten Therapiestrategien Warfarin bzw. Phenprocoumon. Insbesondere bei Patienten mit höherem ischämen Schlaganfallrisiko, mit höheren Blutungsrisiken und mit eingeschränkter Nierenfunktion.

Interessenkonflikte Es bestehen keine Interessenkonflikte.
Open Access Dieser Artikel unterliegt den Bedingungen der Creative Commons Attribution License. Dadurch sind die Nutzung, Verteilung und Reproduktion erlaubt, sofern der/die Originalautor/en und die Quelle angegeben sind.

\section{Literatur}

1. Höhler H. Vorhofflimmern, Risikostratifizierung, Therapie und Prophylaxe. Med Klin. 2005;100(10):636-49.

2. Sanoski CA. Prevalence, pathogenesis, and impact of atrial fibrillation. Am J Health-Syst Pharm. 2010;67 Suppl 5:11-6.

3. Brüggenjürgen B, Reinhold T, McBride D, et al. Vorhofflimmern - epidemiologische, ökonomische und individuelle Krankheitsbelastung. Dtsch Med Wochenschr. 2010;135:21-5.

4. Samardhi H, Santos M, Denman R, et al. Current management of atrial fibrillation. Aust Prescriber. 2011;34(4):100-4.

5. Hart RG, Pearce LA, Koudstaal PJ. Transient ischemic attacks in patients with atrial fibrillation: implications for secondary prevention: the European atrial fibrillation trial and stroke prevention in atrial fibrillation III trial. Stroke. 2004;35(4):948-51.

6. Hart RG, Pearce LA, Aguilar MI. Meta-analysis: antithrombotic therapy to prevent stroke in patients who have nonvalvular atrial fibrillation. Ann Intern Med. 2007;146(12):857-67.

7. Perrey M, Erbel R. Antikoagulation. Herz. 2012;37(4):407-15.

8. Norgard NB, DiNicolantonio JJ, Topping TJ, et al. Novel anticoagulants in atrial fibrillation stroke prevention. Ther Adv Chron Dis. 2012;3(3):123-36.

9. Granger CB, Alexander JH, McMurray JJ, et al. Apixaban versus Warfarin in patients with atrial fibrillation. N Engl J Med. 2011;365(11):981-92.

10. Connolly SJ, Eikelboom J, Joyner C, et al. Apixaban in patients with atrial fibrillation. N Engl J Med. 2011;364(9):806-17.

11. Reinhold T, Lindig C, Willich SN, et al. The costs of atrial fibrillation in patients with cardiovascular comorbidities-a longitudinal analysis of German health insurance data. Europace. 2011;13(9):1275-80.

12. Lindig C, Brüggenjürgen $B$, Willich $S$, et al. Die Kosten des Schlaganfalls - eine Längsschnittanalyse. PharmacoEconomics Ger Res Artic. 2010;8(2):97-107.

13. Gage BF, Cardinalli AB, Owens DK. The effect of stroke and stroke prophylaxis with Acetylsalicylsäure or Warfarin on quality of life. Arch Intern Med. 1996;156(16):1829-36.

14. Milde S. Versorgungsqualität im Krankenhaus, Sekundärprophylaxe, Kosten: Die Versorgung von Schlaganfallpatienten in Hessen. Dissertation, Fakultät für Wirtschaftswissenschaften der Technischen Universität Dresden. 2010.

15. Schwebe M, Marschall P, Fleßa S. Schwerwiegende Arzneimittelnebenwirkungen unter Phenprocoumon: stationäre Kosten der gastrointestinalen Blutungen und Einsparpotentiale. PharmacoEconomics - Ger Res Artic. 2012;10(1):17-28.

16. Kristiansen C, Lassen JF, Dahler-Eriksen BF, et al. Evaluation of a simple dosage scheme for transition from phenprocoumon to warfarin in oral anti4coagulation. Thromb Res. 2000;98(2):157-63.

17. Tiemann O. Variation in hospitalisation costs for acute myocardial infarction-a comparison across Europe. Health Econ. 2008;17 Suppl 1:33-45.

18. Liste R. Editio Cantor. 2013.

19. Lee S, Mullin R, Blazawski J, et al. Cost-effectiveness of Apixaban compared with Warfarin for stroke prevention in atrial fibrillation. PLoS ONE. 2012;7. e47473. doi:10.1371/journal.pone. 0047473. http://www.plosone.org/article/info\%3Adoi\%2F10. 1371\%2Fjournal.pone.0047473 Abgerufen am 20.10.2012

20. Bundesamt für Statistik - Sterbetafeln 2008/2010 aus Periodensterbetafeln. https://www.destatis.de/DE/Publikationen/ Thematisch/Bevoelkerung/Bevoelkerungsbewegung/ Periodensterbetafeln.html. abgerufen am: 20.11.2012. 
21. Eckman MH, Rosand J, Knudsen KA, et al. Can patients be anticoagulated after intracerebral hemorrhage? A decision analysis. Stroke. 2003;34(7):1710-6.

22. Lee S, Anglade MW, Meng J, et al. Cost-effectiveness of Apixaban compared with Aspirin for stroke prevention in atrial fibrillation among patients unsuitable for Warfarin. Circ Cardiovasc Qual Outcomes. 2012;5(4):472-9.

23. Connolly SJ, Pogue J, Hart RG, et al. Effect of Clopidogrel added to Aspirin in patients with atrial fibrillation. N Engl J Med. 2009;360(20):2066-78.

24. Coleman CI, Straznitskas AD, Sobieraj DM, et al. Costeffectiveness of Clopidogrel plus aspirin for stroke prevention in patients with atrial fibrillation in whom Warfarin is unsuitable. Am J Cardiol. 2012;109(7):1020-5.

25. Fang MC, Go AS, Chang Y, et al. Death and disability from warfarin-associated intracranial and extracranial hemorrhages. Am J Ind Med. 2007;120(8):700-5.
26. Rathore SS, Berger AK, Weinfurt KP, et al. Acute myocardial infarction complicated by atrial fibrillation in the elderly: prevalence and outcome. Circulation. 2000;101(9):969-74.

27. Kamel H, Easton JD, Johnston SC, et al. Cost-effectiveness of apixaban vs warfarin for secondary prevention in atrial fibrillation. Neurology. 2012;79(14):1428-34.

28. Gonzalez-Juanatey JR, Alvarez-Sabin J, Lobos JM, et al. Costeffectiveness of Dabigatran for stroke prevention in non-valvular atrial fibrillation in Spain. Rev Esp Cardiol. 2012;65(10):901-10.

29. Davidson T, Husberg M, Janzon M, et al. Cost-effectiveness of dabigatran compared with Warfarin for patients with atrial fibrillation in Sweden. Eur Heart J. 2012;34(3):177-83.

30. Amin A, Stokes M, Wu N, et al. Estimated medical cost reductions associated with Apixaban in real world patients with non-valvular atrial fibrillation. J Med Econ. 2013;16(10):1193-202. 